\title{
Deflection Solutions for Concentrated Force on Spherical Shell Wang Fan ${ }^{\mathrm{a}}$, Wang Xing \\ School of Architecture and Civil Engineering, Ningbo University of Technology, \\ Ningbo 315016, P.R. China \\ awyuqi425@163.com
}

Keywords: Deflection Solution, Spherical Shell, Concentrated Force, Shallow Shell

Abstract. The deflection solution of concentrated loads on spherical thin shell has already been widely studied. The solution for a concentrated normal force was first obtained by E.Reissner in 1946 by using the classical theory of shallow shells. This problem was also considered by S.P.Timoshenko(1946), Stanislaw Lukasiewicz(1979), W.T.Koiter(1963), W.C.Young (1989) etc. In deriving these solutions, it is assumed that the stresses and displacements are very small at some distance from the loading point. In this paper, the displacement of non-shallow spherical thin shell under a concentrated load has been considered. Through apply the above all kinds of methods, we have calculated the deflections under the point of application of concentrated loads on spherical thin shells with $\mathrm{R} / \mathrm{t}=10 \sim 50$ and $\mathrm{R} / \mathrm{t}=100 \sim 500$. The results are analyzed and all kinds of methods are compared, the calculation precisions and covering ranges of all kinds of methods are discussed.

\section{Introduction}

The action of concentrated loads on spherical thin shell has been widely studied. The solution for a concentrated normal force was first obtained by E.Reissner in 1946 by using the classical theory of shallow shells. This problem was also considered by S.P.Timoshenko(1946), Stanislaw Lukasiewicz(1979), W.T.Koiter(1963), W.C.Young (1989) etc. In deriving these solutions it is assumed that the stresses and displacements are very small at some distance from the loading point. The results are analyzed and all kinds of methods are compared, the calculation precisions and covering ranges of all kinds of methods are discussed.

\section{Deflection solutions}

\section{Timoshenko(1946)}

Timoshenko(1946) take the fact into account that the effect of transverse shear $\mathrm{Q}_{\mathrm{r}}$ on membrane forces can be neglected in the case of a shallow shell and the deflection at the center of such a shell is affected very little by the respective conditions on the outer edge. He considered a shallow shell with a very large radius subjected to a point load $\mathrm{P}$ at the apex $\mathrm{r}=0$ (Figure 1), while the normal displaces $\mathrm{w}$ must be finite at $\mathrm{r}=0$, and $\mathrm{w}$ must vanish for $\mathrm{r}=\infty$. He obtained for the deflection of the shell at the point of the application of the load the value (Figure 1):

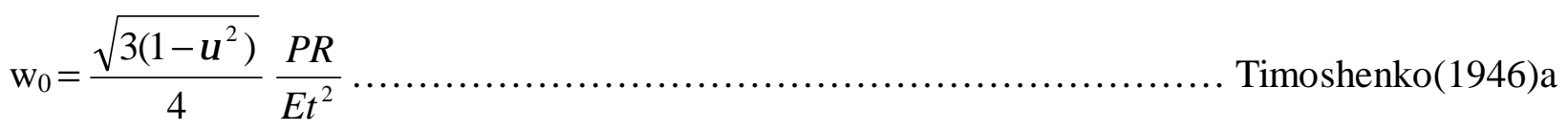

When the central load $\mathrm{P}$ is uniformly distributed over a circular area of a small radius c (Figure 2), the following results hold at the center of the loaded area $r=0$ :

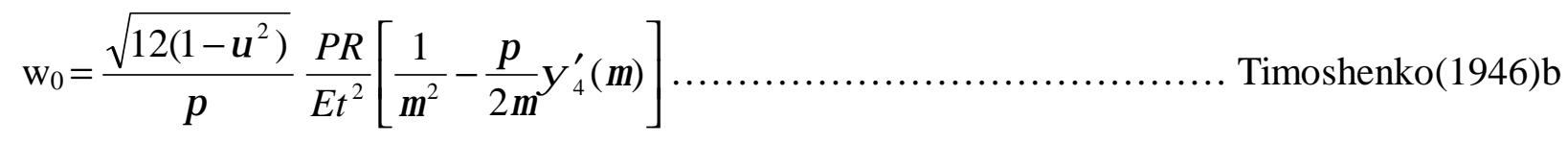


where $\mu=\frac{c}{l}, \quad l=\frac{\sqrt{R t}}{\sqrt[4]{12\left(1-v^{2}\right)}}$; the function $\psi_{4}(\mu)=-(2 / \pi) \operatorname{ker} \mu$, numerical values of the function $\psi_{4}^{\prime}$ are given in Table 86 of Timoshenko(1946).

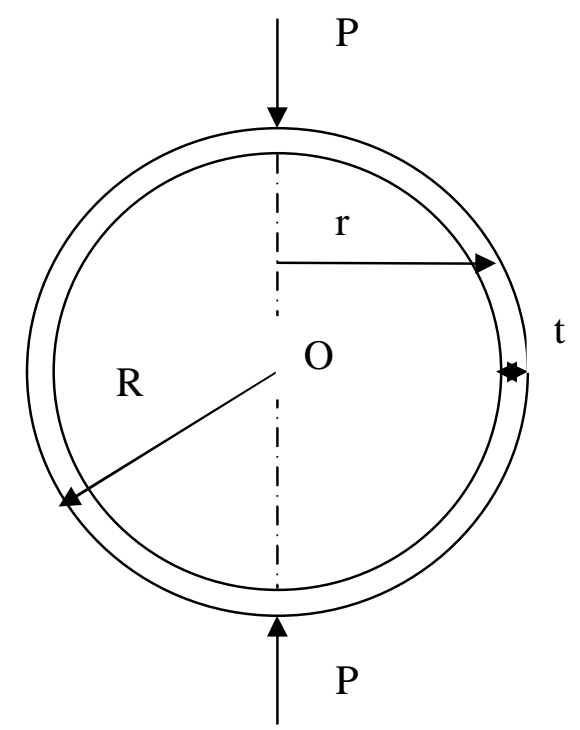

Figure 1: The shell at the point of the load

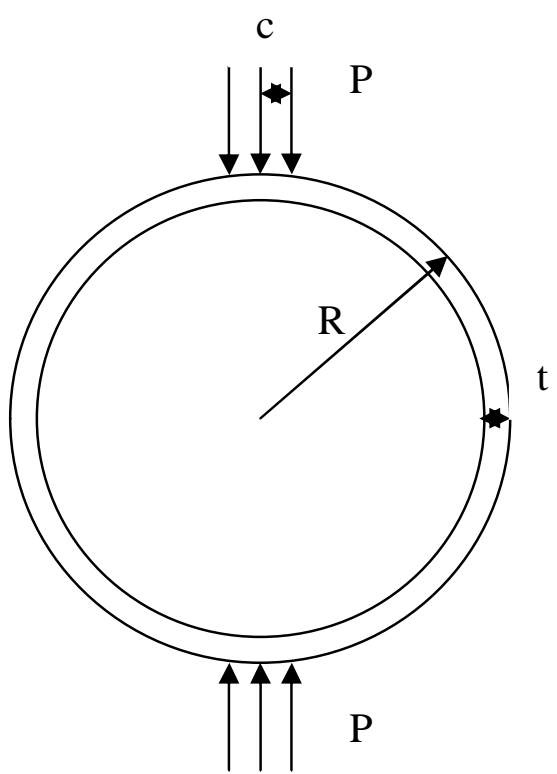

Figure 2: P uniformly distributed

\section{Koiter(1963)}

The non-shallow spherical shell loaded at the vertex has been considered by Koiter(1963) in spherical coordinates, and based on the equations of the theory of non-shallow spherical shell. Koiter obtained the following expression for the normal deflection under the point of application of the load P (Figure 1):

$$
\mathrm{w}_{0}=\frac{\sqrt{3\left(1-v^{2}\right)}}{4} \frac{P R}{E t^{2}}\left[1+\frac{2(1+v)}{\pi \lambda^{2}}\left(\ln \lambda+\gamma_{0}-1+\frac{\ln 2}{2}\right)+\frac{4}{3 \pi \lambda^{2}}+O\left(\lambda^{-3}\right)\right] \ldots \ldots . . . \text { Koiter(1963) }
$$

where

$$
\lambda^{2}=\sqrt{3\left(1-v^{2}\right)} \frac{R}{t} ; \gamma_{0} \text { is Euler's constant, } \gamma_{0}=0.5772
$$

\section{Lukasiewicz(1979)}

Lukasiewicz(1979)[3] also obtained an approximate formula for the displacement of non-shallow shell under a concentrated load P (Figure 1):

$$
\mathrm{w}=\frac{\sqrt{3\left(1-v^{2}\right)}}{4} \frac{P R}{\pi E t^{2}}\left[\pi-4(1+v) k_{R} \ln \sqrt{2 k_{R}}+2 k_{R}+\left(r^{2}-4(\eta-\varepsilon)\right)\left(\ln \frac{r}{2}+\gamma_{0}\right)-r^{2}-\eta\right]
$$

where

$$
k_{R}=\frac{l^{2}}{R^{2}} ; \eta=\frac{t^{2}}{5(1-v) l^{2}} ; \varepsilon=\frac{v t^{2}}{10(1-v) l^{2}}
$$

However, the equation cannot be used to calculate the deflection at those points of the shell that are very close to the point of application of the load $(r<t)$. If we neglect the effect of transverse shear deformation, we can obtain the following expression for the normal deflection under the point 
of application of the load P (Figure 1):

$$
\mathrm{w}_{0}=\frac{\sqrt{3\left(1-v^{2}\right)}}{4} \frac{P R}{\pi E t^{2}}\left[\pi-4(1+v) k_{R} \ln \sqrt{2 k_{R}}+2 k_{R}-\eta\right\rfloor \ldots \ldots \ldots \ldots \ldots \ldots \text { Lukasiewicz(1979)a }
$$

Lukasiewicz(1979) also gave an expression for the normal deflection under the point of application of the load $\mathrm{P}$ when the central load $\mathrm{P}$ is uniformly distributed over a circular area of a small radius c (Figure 2):

$$
\begin{aligned}
& \mathrm{w}_{0}=\frac{\sqrt{12\left(1-v^{2}\right)}}{\pi} \frac{P R}{E t^{2}}\left[\frac{1}{\mu^{2}}+\frac{1}{\mu} \operatorname{ker}^{\prime}(\mu)-\frac{1+v}{2} k_{R} \ln \sqrt{2 k_{R}}-\frac{k_{R}}{4}\right] \\
& +\frac{3}{5 \pi}(1+v)(2-v) \frac{P}{E t}\left[\operatorname{ker} \mu+\frac{\mu \text { ker }^{\prime} \mu}{2(2-v)}\right]
\end{aligned}
$$

Lukasiewicz(1979)b

\section{Young(1989)}

Young(1989) gave a simple formula for partial spherical shell with any edge support and load $\mathrm{P}$ concentrated on small circular area of radius $\mathrm{c}$ at pole. The deflection does not include any deflection due to the edge supports or membrane stresses remote from the loading.

The deflection under the center of the load P (Figure 2):

$$
\mathrm{w}_{0}=A \frac{P R \sqrt{1-v^{2}}}{E t^{2}}
$$

where $A$ is a numerical coefficient that depends upon $\mu$ and its values can be obtained from Table 30 of Young(1989).

The deflections under the point of application of bcalbad $P$ R $/=10^{\sim} 50$ )

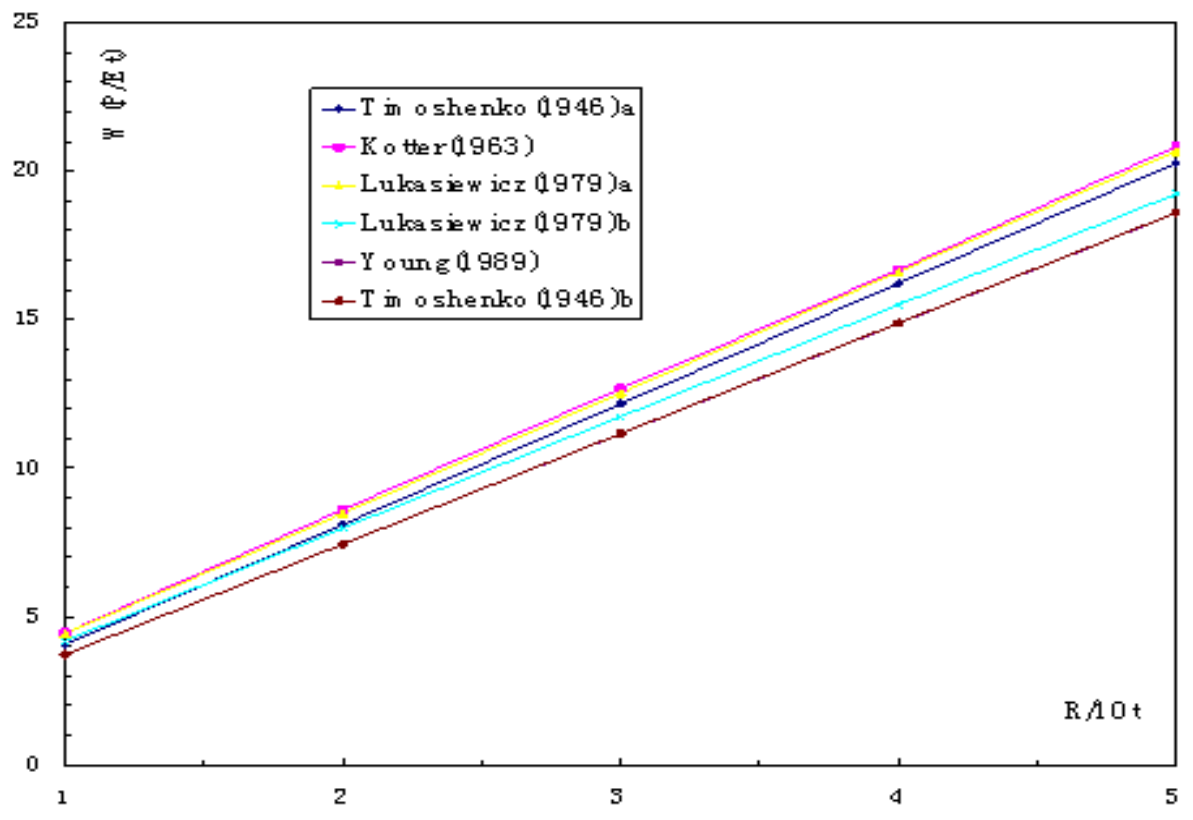

Figure 3: Comparison of solutions for the deflections under the point of application of local load $\mathrm{P}(\mathrm{R} / \mathrm{t}=10 \sim 50)$ 


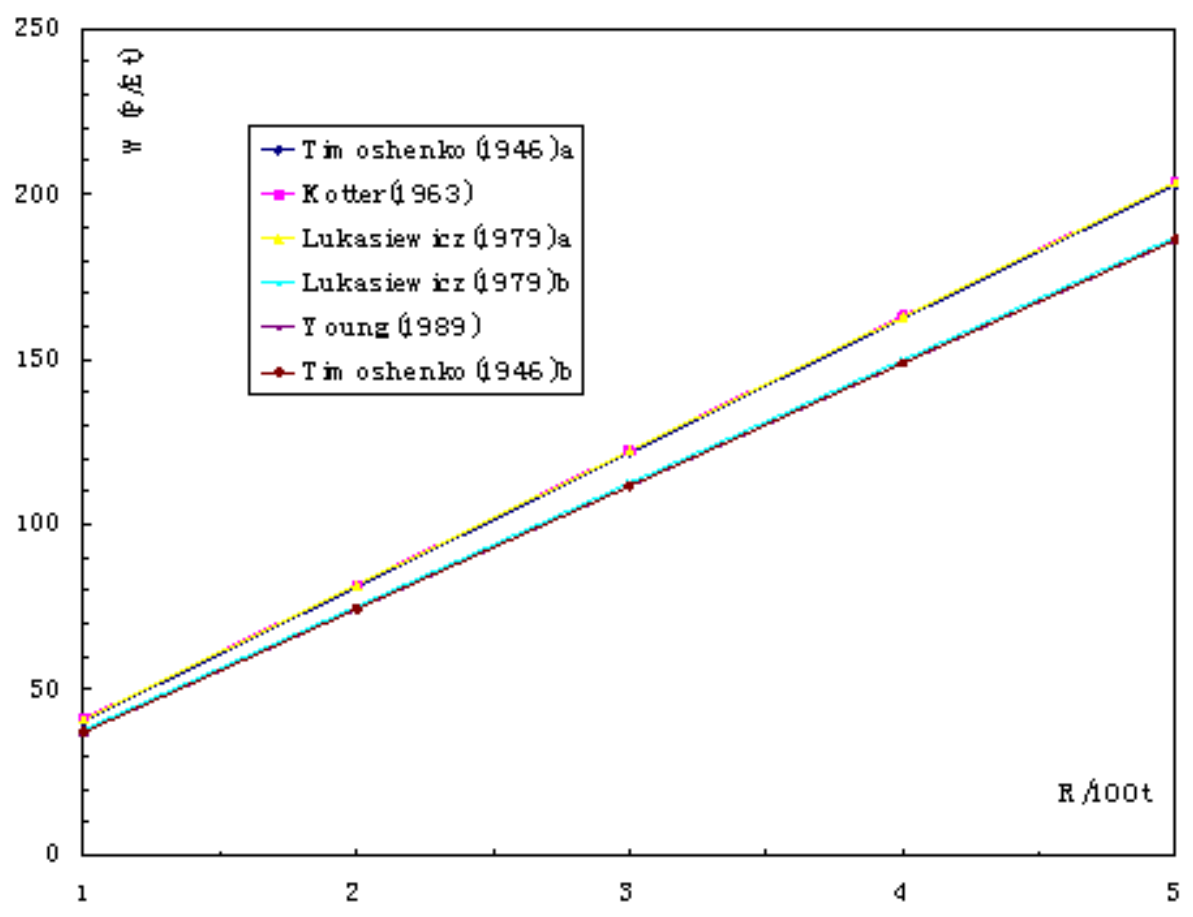

Figure 4: Comparison of solutions for the deflections under the point of application of local load $\mathrm{P}(\mathrm{R} / \mathrm{t}=100 \sim 500)$

\section{Comparison of solutions}

Appling the above all kinds of methods, we have calculated the deflections under the point of application of concentrated loads on spherical shells with $\mathrm{R} / \mathrm{t}=10 \sim 50$ and $\mathrm{R} / \mathrm{t}=100 \sim 500$. The results are shown as the following Figure 3 and Figure 4.

\section{Conclusions}

From the comparision of solutions, we can see that the results of all kinds of solution differ little, specially for $\mathrm{R} / \mathrm{t}=100 \sim 500$. The results of Koiter(1963) and Lukasiewicz(1979)a are very close, the results of Timoshenko(1946)b and Young(1989) are very close too. The solution of Koiter(1963) is most precise, the solution of Young(1989) differ from Koiter(1963) furthest, but the formula is simplest.

\section{References}

[1]. S.P.Timoshenko, Theory of plates and shells, McGraw-Hill, 1946, pp.561.

[2]. W.T.Koiter, A spherical shell under point loads at its poles, Advances in Appl.Mech., Prager Anniversary Volume, 1963,pp.155-169.

[3]. Stanislaw Lukasiewicz, Local loads in plates and shells, PWN-POLISH SCIENTIFIC PUBLISHERS, 1979, pp285, pp289.

[4]. W.C.Young, Roark's formulas for stress and strain, McGraw-Hill, 1989, pp546. 